\author{
Marquette University \\ e-Publications@Marquette
}

2020

\title{
Adiabatic Trajectory Approximation within the Framework of Mixed Quantum/Classical Theory
}

Bikramaditya Mandal

Marquette University

Alexander Semenov

Marquette University

Dmitri Babikov

Marquette University, dmitri.babikov@marquette.edu

Follow this and additional works at: https://epublications.marquette.edu/chem_fac

Part of the Chemistry Commons

\section{Recommended Citation}

Mandal, Bikramaditya; Semenov, Alexander; and Babikov, Dmitri, "Adiabatic Trajectory Approximation within the Framework of Mixed Quantum/Classical Theory" (2020). Chemistry Faculty Research and Publications. 1016.

https://epublications.marquette.edu/chem_fac/1016 
Marquette University

e-Publications@Marquette

\section{Chemistry Faculty Research and Publications/College of Arts and Sciences}

This paper is NOT THE PUBLISHED VERSION.

Access the published version via the link in the citation below.

Journal of Physical Chemistry A, Vol. 124, No. 47 (2020): 9877-9888. DOl. This article is (C) American Chemical Society and permission has been granted for this version to appear in ePublications@Marquette. American Chemical Society does not grant permission for this article to be further copied/distributed or hosted elsewhere without the express permission from American Chemical Society.

\section{Adiabatic Trajectory Approximation within the Framework of Mixed Quantum/Classical Theory}

Bikramaditya Mandal

Chemistry Department, Wher Chemistry Building, Marquette University, Milwaukee, Wisconsin Alexander Semenov Chemistry Department, Wher Chemistry Building, Marquette University, Milwaukee, Wisconsin Dmitri Babikov

Chemistry Department, Wher Chemistry Building, Marquette University, Milwaukee, Wisconsin 


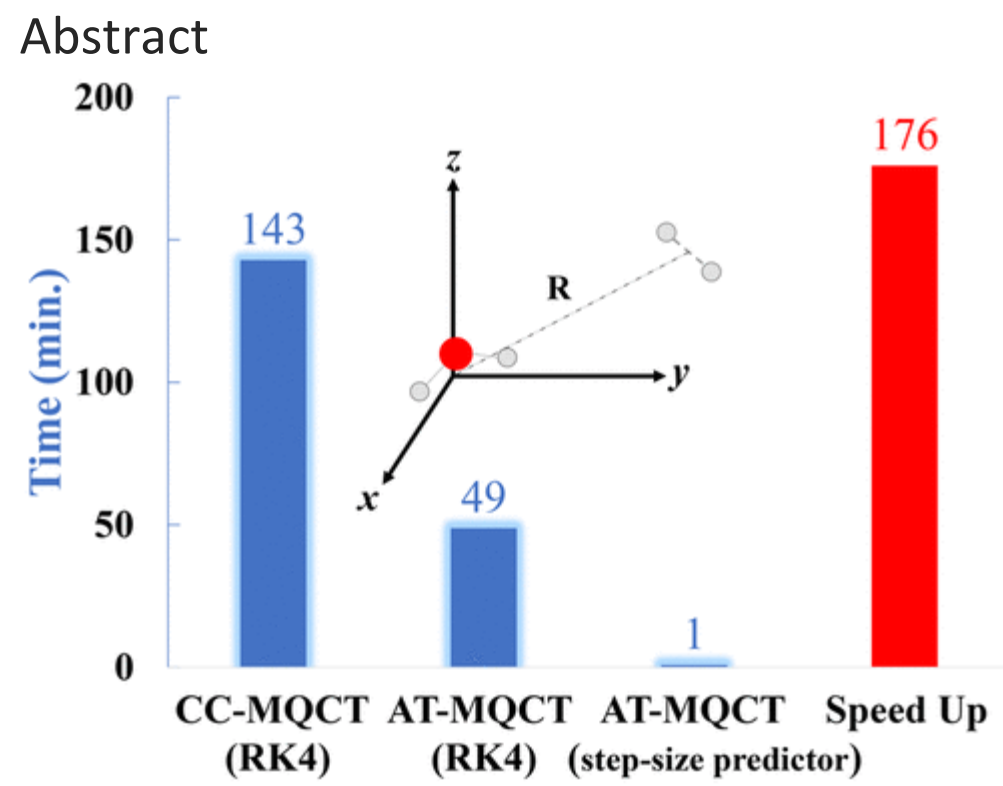

A hierarchy of approximate methods is proposed for solving the equations of motion within a framework of the mixed quantum/classical theory (MQCT) of inelastic molecular collisions. Of particular interest is a limiting case: the method in which the classical-like equations of motion for the translational degrees of freedom (scattering) are decoupled from the quantum-like equations for time evolution of the internal molecular states (rotational and vibrational). In practice, trajectories are precomputed during the first step of calculations with driving forces determined solely by the potential energy surface of the entrance channel, which is an adiabatic trajectory approximation. Quantum state-to-state transition probabilities are computed in the second step of calculations with an expanded basis and very efficient step-size adjustment. Application of this method to $\mathrm{H}_{2} \mathrm{O}+$ $\mathrm{H}_{2}$ rotationally inelastic scattering shows a significant computational speedup by 2 orders of magnitude. The results of this approximate propagation scheme are still rather accurate, as demonstrated by benchmarking against more rigorous calculations in which the quantum and classical equations of motion are held coupled and against the full-quantum coupled-channel calculations from the literature. It is concluded that the AT-MQCT method (the adiabatic trajectory version of MQCT) represents a promising tool for the computational treatment of molecular collisions and energy exchange.

\section{Introduction}

Inelastic collisions of molecules with atomic gasses(1-4) with other gas-phase molecules(5-9) or with solid surfaces(10-15) lead to the energy exchange between translational, rotational, and vibrational degrees of freedom, which is a fundamentally important phenomenon in the field of chemical physics. On the applied side, the inelastic molecular collisions play critical roles in many processes that span a huge range of sizes and time scales from the man-made microtraps(16-19) to the galaxy and extragalactic sizes.(20-24) Therefore, theoretical prediction of inelastic cross sections has been and still is actively pursued, including practical applications of well-known codes to more and more complex molecular processes, $(3,4,18,25-27)$ development of new computational tools, (28-31) and exploration of new theoretical methodologies.(32-37) 
In the last decade, we developed the mixed quantum/classical theory of inelastic molecular scattering $22,38-42)$ and implemented this method in a recently released ready-to-use code named MQCT.(31) Our method is approximate, but it appears to work well in a wide range of collision regimes and for a broad variety of molecular systems. $(22,41,43-45)$ It offers significant computational advantages both in the overall CPU cost required to solve the inelastic scattering problem (due to a simplified physics) and in the user's wall-clock time (enabled by efficient massive parallelization).

Within the framework of MQCT, the internal molecular degrees of freedom are treated quantum mechanically using the time-dependent Schrödinger equation. Therefore, this method takes into account many quantum features of molecular rotations and vibrations, such as state quantization and zero-point energy preservation,(46) symmetry restrictions on allowed and forbidden transitions,(31) propensities of state-to-state transition cross sections,(36) quantum interference effects,(47) and several other quantum features that we keep investigating. At the same time, the translational motion of colliding partners responsible for their scattering in space is treated classically within the Ehrenfest mean-field trajectory approach. $(48,49)$ This simplification was found to work well even for the lightest collision partners such as the $\mathrm{He}$ atom and $\mathrm{H}_{2}$ molecule in a broad range of collision energies. $(31,40,43,45)$ Since the quantum treatment of scattering is avoided, the mixed quantum/classical method enables an affordable computational treatment of heavier collision partners and/or at higher collision energies, the limits when the standard full-quantum methods are not affordable computationally anymore.

In the last few years, our efforts were focused mainly on proving to ourselves and to the community that the mixed quantum/classical methodology can be sufficiently accurate and thus practically useful. Namely, we demonstrated that, for many molecules, the results of MQCT calculations become nearly identical to the full-quantum results in the range of high collision energies, $(38,40,42)$ which is a theoretically important limiting case and a practically important collision regime. Moreover, we showed that the results of MQCT remain reasonably accurate even at low scattering energies near the excitation threshold,(31) which historically was thought of as a quantum scattering regime, not amenable to any semi-classical treatment. At this point, it becomes clear that MQCT represents a generally reliable method with a potential of becoming a practical alternative to the full quantum description of molecular inelastic scattering (except, perhaps, in the ultracold physics conditions).

One interesting feature of the MQCT formalism is that it includes the Coriolis coupling effect in a mixed quantum/classical fashion through transitions between the (quantum) states with different projections of the molecular angular momentum that are driven by the (classical) orbital angular momentum of the relative motion of collision partners. This most rigorous version of MQCT calculations was named CC-MQCT by analogy with a well-known quantum coupled-channel (CC) method that is considered to be exact. In addition to CC-MQCT, we also developed and tested an approximate version of MQCT in which the Coriolis coupling is neglected, leading to a simpler set of equations of motion propagated at a reduced numerical cost. This is a mixed quantum/classical analog of a well-known coupled-states (CS) approximation,(50-54) and therefore, we named our approximate MQCT version as CS-MQCT.(40) We found that it gives acceleration by an order of magnitude, which is quite attractive. Unfortunately, we also found that, for some molecules, the results of CS-MQCT deviate appreciably from the results of more rigorous CC-MQCT and more so at low collision energies.(40) Again, this is analogous to the 
performance of the full-quantum, coupled-states method that is known to be less reliable at lower collision energies.

Therefore, it makes sense to examine other alternative methods for the computational treatment of inelastic molecular scattering (and for the description of rotational-vibrational-translational energy exchange in general) still within the MQCT framework but such that solves the problem in an approximate way and hopefully at a fraction of the computational cost. At the moment, this territory is largely unexplored, and we envision a development of a hierarchy of approximate methods of solving the MQCT equations of motion.

In this methodological paper, we introduce one such option that we want to name AT-MQCT where the prefix stands for the adiabatic trajectory version of MQCT. This approximation is specific to the time-dependent mixed quantum/classical theory, and thus it does not have any direct quantum analogue to our best knowledge. In this method, the classical and quantum equations of motion are decoupled in a sense by conducting MQCT calculations in two consecutive steps. During the first step, the molecular basis size is restricted to the degenerate states of the initial rotational channel only (with different projections of the molecular angular momentum). This information is recorded and used during the second run to propagate the quantum equations of motion along this pre-computed adiabatic path. The Coriolis-like coupling terms are included in both steps of such calculations, which preserves an important physical effect in contrast to the CS approximation where it is entirely neglected.

In the Theory section, we review the formalism of MQCT and introduce the AT-MQCT version of the method. In the section Results, we apply this theory to the $\mathrm{H}_{2} \mathrm{O}+\mathrm{H}_{2}$ system and demonstrate that it produces the systematically reliable and rather accurate results. The new method is benchmarked versus the well-established CC-MQCT version but also versus the full-quantum CC calculations of Dubernet and co-workers for the same system.(24) In the Discussion section, we determine the acceleration due to the adiabatic trajectory approximation, which approaches the factor of $\times 200$, making these mixed quantum/classical calculations relatively cheap. Opportunities for further development of a hierarchy of approximations within MQCT are also discussed. Conclusions are presented in the final section of the paper.

\section{Theory}

Rigorous derivation and detailed discussions of the MQCT equations of motion can be found in the recent literature. $(31,41)$ Here, we give just a brief summary.

\section{MQCT Equations of Motion}

Imagine two collision partners (can be molecule + atom, but generally molecule + molecule) at the beginning of their collision event, separated by distance $R_{\max }$. As time goes, the intermolecular distance $R$ shortens, but then, as molecules scatter and start leaving the interaction region, it increases again. The collision is over when $R$ reaches the initial value of $R_{\max }$. The deflection process can be thought of as rotation of the vector $R \rightarrow$ in the laboratory-fixed center-of-mass reference frame $(31,39,41)$ and is described by the azimuthal angle $\Phi$. Time evolution of these continuous classical variables $R$ and $\Phi$ and of their conjugate momenta $P_{R}$ and $P_{\Phi}$ is described by the classical-like equations of motion:(31,41) 


$$
\dot{R}=\frac{P_{R}}{\mu}
$$

(1)

$$
\dot{\Phi}=\frac{P_{\Phi}}{\mu R^{2}}
$$

(2)

$$
\dot{P}_{R}=-\sum_{n} \sum_{n^{\prime}} e^{i \varepsilon_{n}^{n^{\prime}} t} \sum_{m} \frac{\partial M_{n}^{n^{\prime}}}{\partial R} a_{m n^{\prime}}^{*} a_{m n}+\frac{P_{\Phi}^{2}}{\mu R^{3}}
$$

(3)

$$
\begin{aligned}
& \dot{P}_{\Phi}=-i \sum_{n} \sum_{n^{\prime}} e^{i \varepsilon_{n}^{n^{\prime}} t} \sum_{m} M_{n}^{n^{\prime}} \\
& \times\left[a_{m-n, n^{\prime}}^{*} a_{m n} \sqrt{j^{\prime}\left(j^{\prime}+1\right)-m(m-n)}\right. \\
& +a_{m+n, n^{\prime}}^{*} a_{m n} \sqrt{j^{\prime}\left(j^{\prime}+1\right)-m(m+1)} \\
& -a_{m n^{\prime}}^{*} a_{m-1, n} \sqrt{j(j+1)-m(m-1)} \\
& \left.-a_{m n^{\prime}}^{*} a_{m-1, n} \sqrt{j(j+1)-m(m+1)}\right] / 2
\end{aligned}
$$

(4)

In these formulas, $\varepsilon_{n}^{n \prime}$ describes energy differences between the initial (lower index) and the final (upper index) internal states of the system, say rotational or ro-vibrational states with energies $E_{n}$ and $E_{n}$. Index $m$ labels projections of the total molecular angular momentum $j$ (often called $j_{12}$ ) onto the molecule-molecule axis $\vec{R}$. Summation in eqs 3 and 4 is over all quantum states of the system. Time evolution of probability amplitudes, $a_{m n}(t)$, for these molecular quantum states (rotational and ro-vibrational) is described in the body-fixed reference frame, tied to the moleculemolecule vector $\vec{R}$, and is given by the following quantum-like system of coupled equations:(31,41)

$$
\begin{aligned}
& \dot{a}_{\mathrm{mn}}=-i \sum_{n^{\prime}} e^{i \varepsilon_{n}^{n^{\prime}} t} M_{n^{\prime}}^{n} a_{m n^{\prime}} \\
& -i \dot{\Phi}\left[a_{m-1, n} \sqrt{j(j+1)-m(m-1)}\right. \\
& \left.+a_{m+1, n} \sqrt{j(j+1)-m(m+1)}\right] / 2
\end{aligned}
$$

(5) 
The second term in eq 5 describes transitions between the rotational states with $\Delta m= \pm 1$, driven by the classical orbital angular velocity, $\Phi$, which is the Coriolis coupling effect. The first term in eq 5 includes the potential coupling matrix $M_{n}^{n \prime}$ with real-valued, time-independent elements that depend on $R$ and $m$ (these dependencies are omitted in the equations above for transparency). Efficient methodologies for generation of the initial conditions for MQCT trajectories and for constructing cross sections from the final values of probability amplitudes, $a_{m n}$, have been discussed in the recent literature and will not be repeated here.(22,31,39,41,47)

\section{Adiabatic Trajectory Approximation}

Formulas 1-5 represent a system of coupled differential equations of the first order. Note that the classical system of eqs $1-4$ and the quantum system of eq 5 contain the same variables and thus are coupled. In the most rigorous MQCT treatment, they are propagated in time all together. Such a straightforward approach was implemented in the released version of MQCT.(31)

Although the mixed quantum/classical treatment is more affordable than the full quantum treatment, there are still molecular systems and collision conditions where even the MQCT calculations become computationally demanding. Let us consider the $\mathrm{H}_{2} \mathrm{O}+\mathrm{H}_{2}$ system covered in this paper. Assume that, in the basis for para- $\mathrm{H}_{2} \mathrm{O}$ molecule, we want to include the lowest 45 rotational states with the upper state $j_{1}=8$ at $1442.095 \mathrm{~cm}^{-1}\left(k_{a}=6, k_{c}=2\right)$ and the largest value of $j_{1}=11$ at $1332.166 \mathrm{~cm}^{-1}\left(k_{a}=1, k_{c}=\right.$ 11), whereas for the para- $\mathrm{H}_{2}$, we want to include two rotational states with the upper state $j_{2}=2$ at $365.118 \mathrm{~cm}^{-1}$. This gives us 90 energetically non-degenerate asymptotic rotational states overall in the basis for the $\mathrm{H}_{2} \mathrm{O}+\mathrm{H}_{2}$ system. Within each of these asymptotic states, the complete basis contains all $j_{12}$ states in the range $\left|j_{1}-j_{2}\right| \leq j_{12} \leq j_{1}+j_{2}$ that here we will call the channels. In the example above, the largest value of the total molecular angular momentum is $j_{12}=13$, and there are 264 channels overall. Within each molecular channel, there are $2 j_{12}+1$ projection states with different values of $m$. Altogether, this gives 3486 unique quantum states in the basis and a huge state-to-state transition matrix $M_{n}^{n \prime}(R ; m)$. In the MQCT code,(31) zero matrix elements are identified and excluded, but still, for the example above, the number of unique non-zero matrix elements included in the triplesummation of eqs 3 and 4 is 336,595, which represents a numerical challenge since these summations need to be re-computed at each time step (several times) along each trajectory. Indeed, from the extensive profiling of MQCT calculations, we found that over $50 \%$ of the total numerical effort was used to compute the right-hand sides of eqs 3 and 4 and over $65 \%$ to propagate eqs 3 and 4 despite the fact that these are just two equations in a huge system of coupled differential equations (6976 equations in total in our example; out of which, 4 are for the classical degrees of freedom, while 6972 are for the real and imaginary parts of 3486 quantum probability amplitudes).

It is also important to realize that, when computed, the triple summations in the right-hand sides of the classical equations produce just two numbers, the average gradient for eq 3 and the average torque for eq 4 . How sensitive are these average values to the contribution of each term in the sum? Do we really need to take into account all these terms? Can we neglect the contributions of some terms? Which terms contribute more and thus must be retained? Clearly, more than one truncation scenario is possible here depending on the answers to these questions, and we will discuss various alternatives in detail further below (see Discussion). Here, we will propose and test a simple solution that, in a sense, goes to the extreme. It is discussed next. 
It is clear that the largest contributions to the right-hand sides of eqs 3 and 4 will normally come from the most populated states, those with larger values of probability amplitudes, $a_{m n}(t)$. For many molecular systems and many collision conditions, the inelastic transition probabilities are relatively small, and thus the survival probability (in the initial channel) is dominant. Therefore, one simple way to truncate the sum in eqs 3 and 4 is to retain only the states of the initial channel. This can include all the degenerate $m$ states since the number of such states within one channel is usually manageable, $2 j_{12}+1$. With this choice, the MQCT trajectories will be driven by the potential energy surface associated with one quantum channel only (the initial channel), rather than by the average Ehrenfest potential. Such trajectories are not sensitive to excitation or quenching of the initial state and, strictly speaking, are not the mean-field trajectories anymore. These are adiabatic trajectories, which suggests the name AT-MQCT for this approximation.

Using truncation of the right-hand sides in eqs 3 and 4, one could still hold the overall system of MQCT equations (eqs 1-5) coupled as before to propagate in time all the equations of motion together, or, alternatively, one could go beyond just truncation and, following the spirit of the adiabatic ansatz, try to completely decouple the propagation of four classical equations of motion, eqs 1-4, from the huge system of quantum coupled equations, eq 5 . In practice, this can be implemented as follows:

1. In the first run, propagate all MQCT equations of motion rigorously coupled as before but using an adiabatic basis that includes only $2 j_{12}+1$ states (labeled by $m$ in eqs $1-$ 5 above) of the initial quantum channel. Since the basis is small, such MQCT calculations would be trivial for any molecular system without any other approximations. The existing MQCT code can be used without modifications. These would be adiabatic MQCT calculations since no rotational excitation or quenching of the internal molecular states is possible within such a basis, but the goal is to record the trajectory data as a function of time and to use this information during the second run. For example, one can record all classical variables: $R(t), \Phi(t), P_{R}(t)$ and $P_{\Phi}(t)$, or one can record only $R(t)$ and $\dot{\Phi}(t)$ that are specifically needed in the second run. As you will see, it is also beneficial to record average potential $V(t)$ along the trajectory.

2. In the second run, the basis is increased to the desired size in order to describe all the state-to-state excitation and quenching transitions (e.g., 90 states in the $\mathrm{H}_{2} \mathrm{O}+$ $\mathrm{H}_{2}$ example above), but now only the quantum system of coupled equations is propagated in time, using eq 5 , to determine the evolution of probability amplitudes, $a_{m n}(t)$. The value of $R$ for the state-to-state transition matrix $M_{n}^{n \prime}(R ; m)$ and the value of $\dot{\Phi}$ for the Coriolis coupling (in the first and second terms of eq 5 , respectively) are "borrowed" from the adiabatic trajectory data saved during the first run (e.g., using a 1D spline along the trajectory, which is a computationally cheap trick). Only minor technical modifications to the code are needed.

Of course, such decoupling of classical and quantum degrees of freedom in the AT-MQCT method is an approximation that needs to be tested by numerical simulations (see the next section). One downside of this assumption is that the total energy of the system is not conserved anymore. However, the norm of the wavefunction is still conserved and can be monitored to ensure the convergence of numerical integration. One important advantage of this method, compared to the CS-MQCT approximation, is 
that the Coriolis coupling terms in eqs 4 and 5 are included in both steps of the AT-MQCT calculations: in the first adiabatic step as well as in the second non-adiabatic step.

\section{Adiabatic Step-Size Predictor}

The previously released version of MQCT(31) had a fourth-order Runge-Kutta integrator built in for the propagation of the total system of coupled equations: the classical eqs 1-4 and the quantum eq 5 altogether. By default, the constant step-size was used with an option of adaptive step-size control from Numerical Recipes.(55) We found, however, that the adaptive step-size control routine gave advantage only for the molecular systems with a long-range interaction potential, such as $\mathrm{H}_{2} \mathrm{O}+$ $\mathrm{H}_{2} \mathrm{O} .(31,36,41)$ For other cases, such as the $\mathrm{H}_{2} \mathrm{O}+\mathrm{H}_{2}$ system considered here, the "black-boxed" stepsize control gave no noticeable increase of performance. However, we realized that the adiabatic trajectory approximation introduced above gives us an opportunity to implement a very efficient method for the variable-step integration of the quantum system of equations, eq 5, propagated during the second step of the AT-MQCT calculations (which is the only costly part of new method). For this, we can take advantage of the $V(t)$ dependence recorded along the adiabatic trajectory in the first step of AT-MQCT calculations.

Indeed, the form of the right-hand side of eq 5 suggests that the time-evolution of probability amplitudes, $a_{m n}(t)$, is driven by the magnitudes of matrix elements, $M_{n}^{n \prime}(R ; m)$, as molecule-molecule separation $R(t)$ first decreases and then increases along the trajectory. Although individual matrix elements, $M_{n}^{n \prime}$, change slightly differently as a function $R$, the potential $V(t)$ recorded during the first step gives us a reasonable prediction of their overall behavior (as they increase from the asymptotic region to the interaction region). Importantly, the numerical error of integration is also expected to depend on the magnitude of matrix elements, $M_{n}^{n \prime}$, and therefore the variable integration step-size $\Delta t$ may be efficiently predicted using the $V(t)$ dependence.

If we keep using the fourth-order Runge-Kutta method in which the integration error $I$ is proportional to $\Delta t^{4}$ and also take into consideration that the numerical error is proportional to the interaction potential $V(t)$, we can write

$$
I=a \cdot \tilde{V} \cdot \Delta t^{4}
$$

where $\alpha$ is a proportionality coefficient that takes care of units and $\tilde{V}$ is the average value of $V(t)$ during the time step $\Delta t$. This average can be computed, for example, by taking $N$ tiny steps, $\tau$, through the interval $\Delta t$ from $t$ to $t+\Delta t$ :

$$
\tilde{V}=\frac{1}{N} \sum_{i=1}^{N} V\left(t+\left[i-\frac{1}{2}\right] \tau\right)
$$


Then, $\Delta t=N \tau$. The goal is to vary $\Delta t$ as $\tilde{V}$ changes along the trajectory in such a way that the numerical error remains constant, for example, does not exceed a specified value of $I_{\max }$. This is achieved by the setting based on eq 6 :

$$
\Delta t=\sqrt[4]{\frac{I_{\max }}{\alpha \cdot \dot{V}}}
$$

(8)

From eqs 6 and 7, it follows that this can be implemented by taking tiny steps $\tau$ along the trajectory, accumulating the predicted value of error

$$
\frac{I}{\alpha}=(N \tau)^{4} \cdot \frac{1}{N} \sum_{i=1}^{N} V(t+[i-1 / 2] \tau)
$$

(9)

and monitoring that, within each time step, it does not exceed $I_{\max } / \alpha$. The value of $I_{\max }$ can be defined by considering a hypothetic trajectory with a very weak interaction such that the potential remains negligibly small at any moment in time: $V(t)<V_{\text {tiny }}$. In this case, the integration of the entire trajectory can be done in one step, $\Delta t=T$ where $T$ is the duration of trajectory (the time $t$ when the moleculemolecule separation reaches $R_{\max }$, also determined in the first step of AT-MQCT propagation). From these considerations and using eq 6 , we obtain

$$
\frac{I_{\max }}{\alpha}=T^{4} \cdot V_{\text {tiny }}
$$

It is more convenient to introduce a unitless measure of precision:

$$
\epsilon=\sqrt[4]{\frac{V_{\text {tiny }}}{U}}
$$

where $U$ is a characteristic value energy, say, the kinetic energy of collision. The values of kinetic energy in typical MQCT calculations vary in the range from 1 to $10,000 \mathrm{~cm}^{-1}$. The typical values of $\epsilon$ are 0.01 and below, say, down to $10^{-5}$. With these definitions, the final formula for the variable time-step is obtained from eqs 8,10 , and 11 :

$$
\Delta t=\epsilon T \cdot \sqrt[r]{\frac{U}{\tilde{V}}}
$$


We checked by extensive calculations and confirmed that this method of step-size prediction works efficiently along the entire trajectory (as it goes from the asymptotic range to the interaction region and back) and for all trajectories in the batch (from the most intense head-on collisions to the more typical side-scattering and forward scattering and the asymptotic trajectories that barely touch the interaction region). Varying one convergence parameter $\epsilon$ permits us to achieve monotonic convergence for all trajectories in the batch, which is very convenient and numerically efficient.

We checked and found that our step-size prediction works for a broad range of collision energies, but the value of $\epsilon$ needs to be adjusted. Namely, at higher energies, the collision is faster, so the time step needs to be reduced, according to $\Delta t \approx 1 / \sqrt{U}$. This relationship occurs simply because $\Delta t=$ $\Delta R / v$ where velocity depends on collision energy as $v=\sqrt{2 m U}$. Thus, if we want the integration to proceed with roughly the same steps through space (through the potential energy surface) at different energies, we have to adjust the time step accordingly. This adjustment is analytic, and we found a simple way to scale the value of $\epsilon$ inside the code (without user's intervention) to take this effect into account. We also found, by trial and errors, that, at the very low collision energies, a higher accuracy is needed, and the value of $\epsilon$ has to be reduced too. Based on our experimentations with the $\mathrm{H}_{2} \mathrm{O}+$ $\mathrm{H}_{2}$ system, we derived an empirical dependence of this effect and have also hardcoded it into the MQCT program. As it stands now, the user can enter one single value of $\epsilon$ that will guarantee the same level of accuracy for all AT-MQCT trajectories at all collision energies. For the $\mathrm{H}_{2} \mathrm{O}+\mathrm{H}_{2}$ system, we observed that, as the value of $\epsilon$ is reduced, all AT-MQCT trajectories converge monotonically. We hope that this behavior is general and plan checking the performance of the adiabatic step-size predictor for other molecular systems in the near future.

To avoid confusion, we would like to stress one more time that this adiabatic predictor scheme is not used to propagate the classical-like equations of motion (eqs 1-4). This method is used only for the quantum-like eq 5 for evolution of probability amplitudes, $a_{m n}(t)$, along the precomputed trajectories. While classical equations of motion include the gradient of potential (the force), the quantum time-dependent Schrödinger equation includes the potential itself. This difference manifests in eqs 3 versus 5 where the right-hand side of the first (classical-like) formula contains gradients of matrix elements, while the right-hand side of the second (quantum-like) formula contains matrix elements themselves.

\section{Results}

In order to benchmark the accuracy and performance of the newly developed approximate AT-MQCT methodology, we carried out a series of calculations for a reasonably complex real molecular system of astrophysical importance, $\mathrm{H}_{2} \mathrm{O}+\mathrm{H}_{2}$. It was studied in the past by various groups, $(24,56)$ and a fair amount of data are available in the literature. In order to make the comparison meaningful, we used the same potential energy surface.(24)

\section{Comparison of AT-MQCT versus CC-MQCT}

The initial distance between the collision partners was set to $R_{\max }=30 a_{0}$, and the maximum impact parameter was selected as $b_{\max }=R_{\max }$ at all collision energies (which automatically defines the maximum value of the orbital angular momentum at each collision energy). The range of collision 
energies covered in this test was from 20 to $7000 \mathrm{~cm}^{-1}$. The rotational basis incorporates 20 asymptotic rotational states of $\mathrm{H}_{2} \mathrm{O}+\mathrm{H}_{2}$ with energies below $500 \mathrm{~cm}^{-1}$, which includes 4 states with hydrogen molecules excited to $j_{2}=2$. For water, the upper rotational state in this test was $4_{40}$, where two subscripts stand for $k_{a}=4$ and $k_{c}=0$, of $j_{1}=4$. The standard CC-MQCT calculations that are expected to serve as a reference and thus need to be perfectly converged were carried out in a standard way using the RK4 integrator with a constant time-step set to a rather small value, $\Delta t=10 \mathrm{a}$. $\mathrm{u}$. Convergence of the approximate AT-MQCT calculations with an adiabatic step-size predictor was also rigorously checked by varying the value of $\epsilon$. The results presented in Figures 1 and 2 are found to be entirely converged when $\epsilon=10^{-3}$.

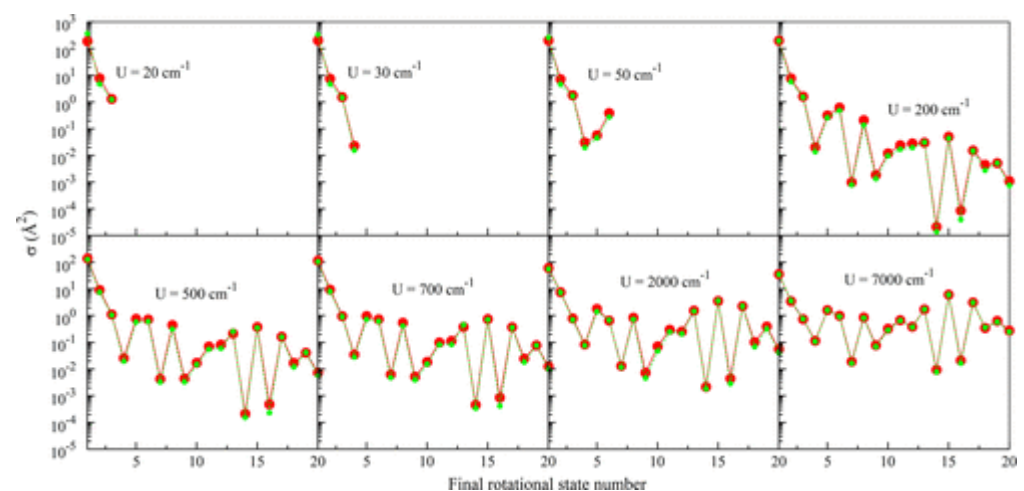

Figure 1. Comparison of results of the new approximate AT-MQCT method (green dots) against the full-coupled CC-MQCT calculations (red symbols) for the initial state $0_{00} \mathrm{O}$ of the $\mathrm{H}_{2} \mathrm{O}+\mathrm{H}_{2}$ system. Final states are listed along the horizontal axis. The values of collision cross sections are plotted along the vertical axis using the log scale. Eight frames correspond to eight values of the kinetic energy as indicated. Twenty rotational states of the basis include both ground and excited rotational states of $\mathrm{H}_{2}$.

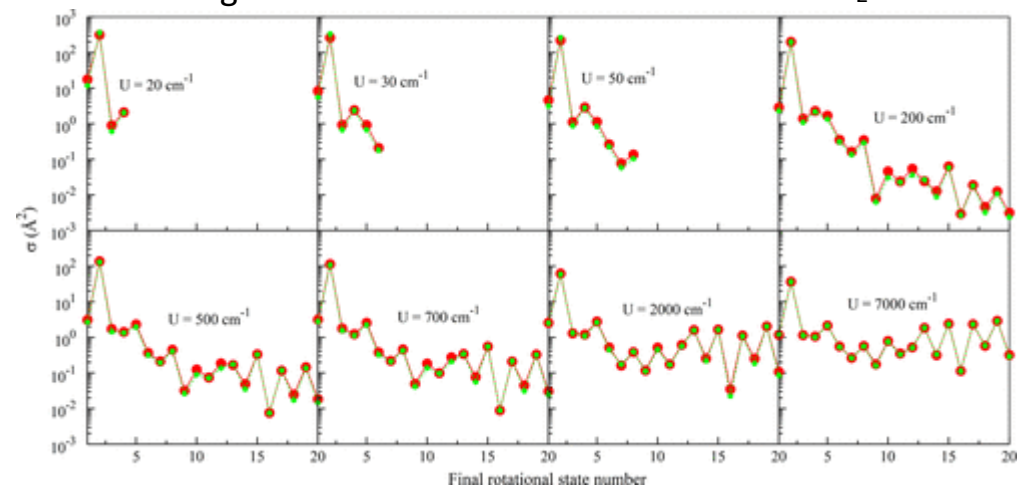

Figure 2. Same as Figure 1 but for the initial state $1_{11} \mathrm{O}$ of the $\mathrm{H}_{2} \mathrm{O}+\mathrm{H}_{2}$ system, which is the first excited rotational state.

First, we looked at the excitations from the ground rotational state of the system, $\mathrm{H}_{2} \mathrm{O}\left(\mathrm{j}_{1}=0\right)+\mathrm{H}_{2}\left(\mathrm{j}_{2}=\right.$ 0 ), which we denote here as $0_{00} 0$. Figure 1 reports cross sections for 20 transitions, including the elastic channel, for eight values of collision energies (one may notice that, at lower collision energies, some channels are closed). One can see that results of the approximate AT-MQCT (green symbols, dashed lines) follow closely the trend of the benchmark CC-MQCT data (red symbols, solid lines) through all transitions and all collision energies systematically. We did not see even one transition when the adiabatic trajectory approximation would fail miserably. It should be emphasized that the values of cross sections vary through 7 orders of magnitude, and still, the results of the approximate AT-MQCT method remain very close to the results of the original CC-MQCT method. At higher energies, the 
agreement is generally better, which is somewhat counterintuitive since one would expect that the error of adiabatic trajectory approximation (single-state) may increase together with possibilities of multiple-state excitations enabled by higher collision energies, but this does not happen. Higher errors are found at lower collision energies when only a few channels are open and for lower rotational states, which is also somewhat unexpected.

Next, we looked at the quenching and excitations of the first excited rotational state of water, $\mathrm{H}_{2} \mathrm{O}\left(j_{1}=\right.$ $\left.1, k_{a}=1, k_{c}=1\right)+\mathrm{H}_{2}\left(j_{2}=0\right)$, which we denote here as $1_{11} 0$. Figure 2 reports cross sections for transitions into the same 20 states of the basis at the same collision energies as in Figure 1 (with all the same convergence parameters chosen). These data again indicate an excellent systematic agreement between the approximate AT-MQCT method (green symbols, dashed lines) and the benchmark CCMQCT method (red symbols, solid lines) through all transitions, all collision energies, and 7 orders of magnitude of cross-section values.

A different viewpoint is obtained by plotting the values of cross sections as a function of collision energy. This is done in Figure 3 for transitions into the five lower energy states of $\mathrm{H}_{2} \mathrm{O}+\mathrm{H}_{2}$, starting from the ground state $0_{00} 0$, and in Figure 4 for the rotationally excited initial state $1_{11} 0$. In these figures, different colors correspond to different transitions. Vertical dashed lines indicate energy thresholds of the individual processes. From Figures 3 and 4, we can see clearly that the results of the adiabatic trajectory approximation do approach monotonically the results of the full-coupled MQCT calculations as the collision energy is increased. Larger deviations are found at lower collision energies, often at threshold. The positions of thresholds are correctly captured by the approximate AT-MQCT method, but the values of cross sections just above the threshold are usually underestimated. For the transitions plotted in Figures 3 and 4, the largest underestimations of this kind are by approximately $40 \%$, found for transitions $0_{00} \rightarrow 1_{11}$ and $1_{11} \rightarrow 2_{02}$ near their thresholds at collision energies 37.158 and approximately $33.001 \mathrm{~cm}^{-1}$, respectively.

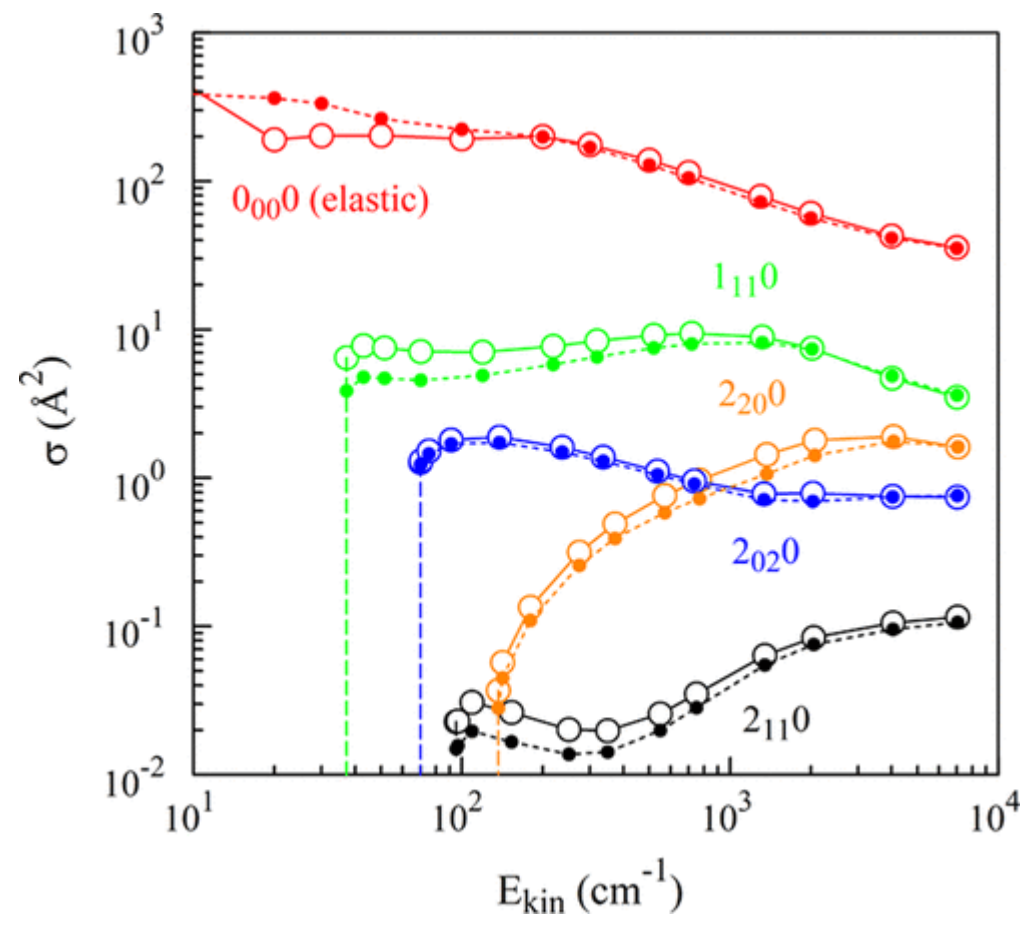


Figure 3. Comparison of results of the new approximate AT-MQCT method (empty circles, solid line) against the full-coupled CC-MQCT calculations (filled symbols, dashed lines) for the initial state $\mathrm{O}_{00} \mathrm{O}$ of the $\mathrm{H}_{2} \mathrm{O}+\mathrm{H}_{2}$ system. The values of collision cross sections are plotted along the vertical axis using the log scale. The kinetic energy of collision (including Billing's correction) is plotted along the horizontal axis. Colors correspond to five different final rotational states as indicated in the picture. Excitation thresholds are shown by the vertical dashed lines.

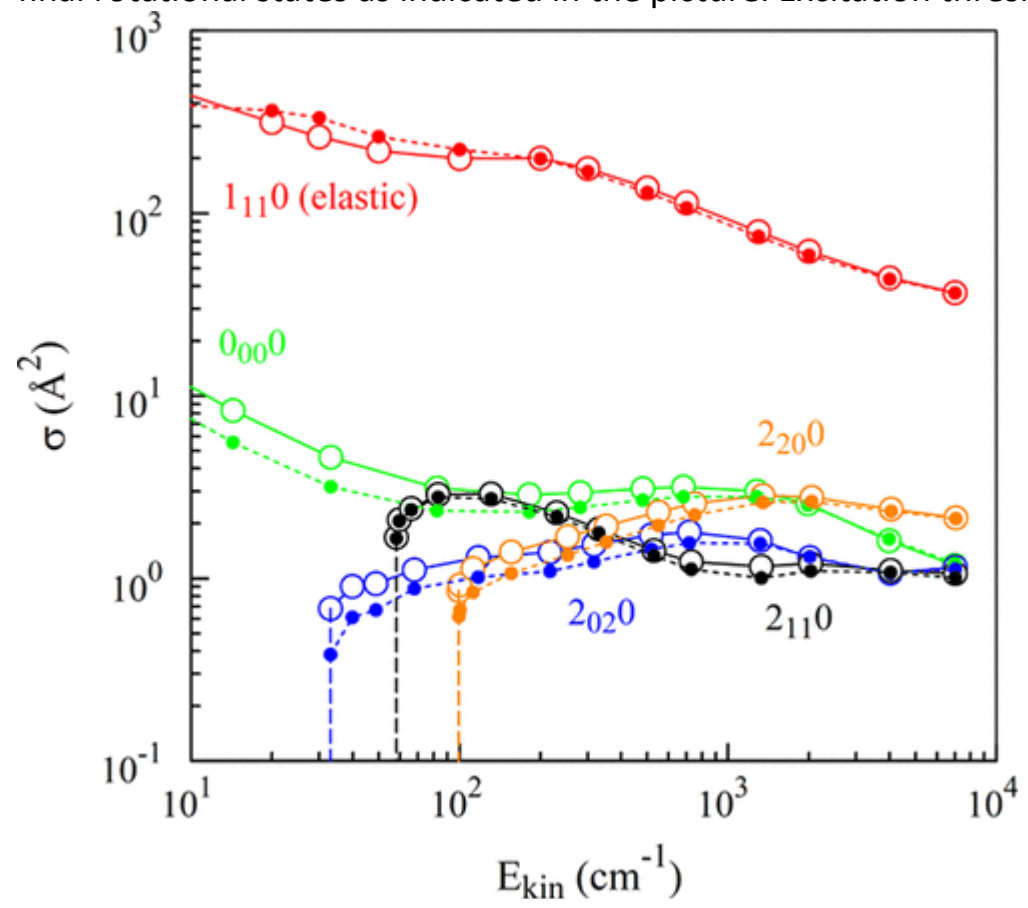

Figure 4. Same as Figure 3 but for the first excited state $1_{11} 0$ of $\mathrm{H}_{2} \mathrm{O}$ as the initial channel.

To summarize, the results of adiabatic-trajectory approximation within the MQCT framework appear to be systematically accurate, particularly at higher collision energies. When it concerns only the trajectory of collision, we do not lose much by restricting the basis size to include only the states of the initial quantum channel. Such an approach is found to be less accurate at the limit of low collision energies, near the process threshold, but this collision regime is often amenable to the full-quantum treatment of scattering, which we discuss next.

\section{Comparison of AT-MQCT versus the Full-Quantum CC Method}

For the $\mathrm{H}_{2} \mathrm{O}+\mathrm{H}_{2}$ system, the results of full-quantum coupled-channel calculations are available from the detailed work of Dubernet and co-workers.(24) In addition to thermal rate coefficients, they also reported the energy dependencies of cross sections for a number of individual state-to-state transitions. Their results are reproduced in Figure 5 (with permission of the author). For the same transitions, we carried out the approximate AT-MQCT calculations. In those, all the convergence parameters were kept as in the previous test, except that the rotational basis size was dramatically increased to match the basis size in the calculations of Dubernet and co-workers at higher collision energies. Namely, 90 asymptotic rotational states of $\mathrm{H}_{2} \mathrm{O}+\mathrm{H}_{2}$ with energies below $1810 \mathrm{~cm}^{-1}$ were included with two states of the hydrogen $\left(j_{2}=0,2\right)$ and the states of water up to 862 . In Figure 5 , four colors correspond to four state-to-state processes in $\mathrm{H}_{2}$, two elastic ones: $0 \rightarrow 0$ (black) and $2 \rightarrow 2$ (blue), one quenching $2 \rightarrow 0$ (green) and one excitation $0 \rightarrow 2$ (red). Three initial states of water are considered: $2_{02}$ (three frames on the left side), $3_{22}$ (three frames in the middle of the picture), and 
$4_{40}$ (three frames on the right side). The final states of water are different for each frame as indicated in the figure caption and include $0_{00}, 1_{11}, 2_{02}, 2_{11}, 3_{31}$, and $5_{24}$.
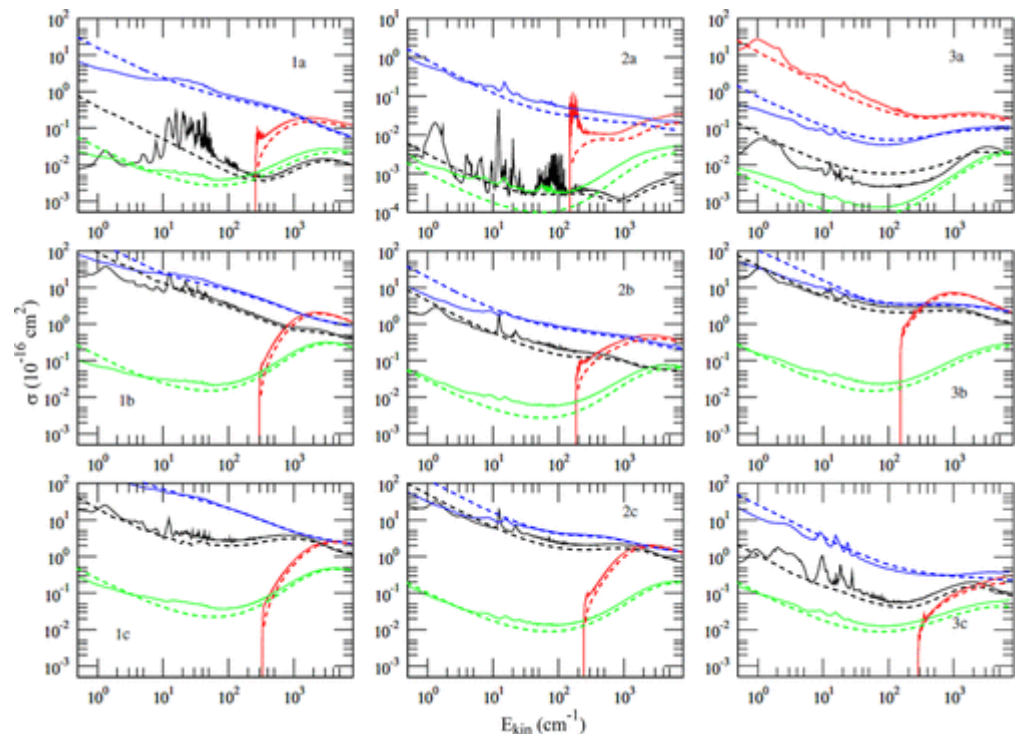

Figure 5. Comparison of results of the new approximate AT-MQCT method (dashed lines) against the fullcoupled CC-MQCT calculations (solid lines) of ref (24) for the $\mathrm{H}_{2} \mathrm{O}+\mathrm{H}_{2}$ system. The values of collision cross sections are plotted along the vertical axis, while the kinetic energy of collision is plotted along the horizontal axis, both in the log scale. Black, red, green, and blue colors are for $0 \rightarrow 0,0 \rightarrow 2,2 \rightarrow 0$, and $2 \rightarrow 2$ transitions in $\mathrm{H}_{2}$, respectively. In $\mathrm{H}_{2} \mathrm{O}$, the transitions are from state $2_{11}$ to states (1a) $0_{00}$, (1b) $1_{11}$, and (1c) $2_{02}$; state $3_{22}$ to states $(2 a) 0_{00},(2 b) 1_{11}$, and (2c) $2_{11}$; and from state $4_{40}$ to states $(3 a) 1_{11}$, (3b) $3_{11}$, and (3c) $5_{24}$.

Overall, Figure 5 contains energy dependencies of 36 transitions, which is a rather comprehensive set of data for the benchmarking of new methods. In all these cases, the results of our AT-MQCT method show very good systematic agreement with the full-quantum results of Dubernet and coworkers.(24) Some differences are present, but they are relatively small and typically vanish at high collision energies. One possible source of small differences at higher energies is likely the fact that a Kyro-type Hamiltonian for the water molecule was used by Dubernet and co-workers, while we employ a simpler rigid-rotor model. Also, given the difficulty of achieving convergence of the full-quantum calculations at high energies, we would cautiously assume that some of these differences may originate in the full quantum calculations too. The AT-MQCT results are expected to be quite reliable in the high-energy regime.

Moreover, it is also quite encouraging that, in the low-collision energy regime, the results of AT-MQCT remain close to the full-quantum results that are considered to be exact. Out of 36 state-to-state transition processes in this figure, we spotted only one transition (frame 1a, dashed vs solid black lines) where the results of AT-MQCT calculations deviate significantly from the full-quantum results: $2{ }_{11} 0 \rightarrow$ $0_{00} 0$ at collision energies below $10 \mathrm{~cm}^{-1}$. Note that this particular process has a number of narrow resonances densely packed in the range of collision energies between 10 and $100 \mathrm{~cm}^{-1}$. These quantum features cannot be described within the AT-MQCT method, which may explain the asymptotic difference observed at very low collision energies in frame 1a of Figure 5. 
Time-Dependent Insight from AT-MQCT

Indeed, quantum resonances are entirely absent in our calculations, but this is expected since the adiabatic trajectory approximation by construct prohibits energy transfer in the first step of AT-MQCT calculations and therefore disables the mechanism of Feshbach resonance formation.(47) If the resonant behavior is present in the low-energy range and happens to be important, then the fullquantum calculations are probably indispensable.

However, there are a number of interesting phenomena where the MQCT results appear to capture the physics correctly. Take a closer look at frame $3 a$, red lines, that describes the $4_{40} 0 \rightarrow 1_{11} 2$ transition. Notice that, among nine processes represented by red lines in Figure 5, this is the only one that has no energy threshold! The value of the cross section keeps increasing as the collision energy is decreased, and our AT-MQCT method (dashed red line) reproduces this behavior very well in comparison with the full-quantum result (solid red line). This particular process can be thought of as a direct energy exchange between the two collision partners: the hydrogen molecule is excited, $0 \rightarrow 2$, while the water molecule is quenched, $4_{40} \rightarrow 1_{11}$. Energy released by one molecule is sufficient to excite the other molecule directly without using the kinetic energy of collision.

Since MQCT offers a useful time-dependent picture, we tried to gain more insight into this quasiresonant energy-exchange process. In Figure 6 , we plotted the values of state populations along a typical AT-MQCT trajectory with impact parameter $b=4.602 a_{0}$, which corresponds to the orbital angular momentum $L=6$ at collision energy $U=56.5 \mathrm{~cm}^{-1}$. The population of the elastic channel (survival probability) corresponds to the initial state $44_{40} \mathrm{O}$ of $\mathrm{H}_{2} \mathrm{O}+\mathrm{H}_{2}$, i.e., hydrogen is in the ground state, whereas water is excited. Figure 6 shows that the population of this state (black) drops quickly as two molecules start colliding then it slightly oscillates right after the collision and finally goes to an asymptotic value close to $\sim 0.8$. The population of the final state $1_{11} 2$ (red in Figure 6 ) shows a roughly opposite trend but is not a mirror image of the initial state population, and the final probability in the $1_{11} 2$ state is only $\sim 5.63 \times 10^{-3}$ (notice the scale factor). Therefore, where does the rest of the initial state population go?

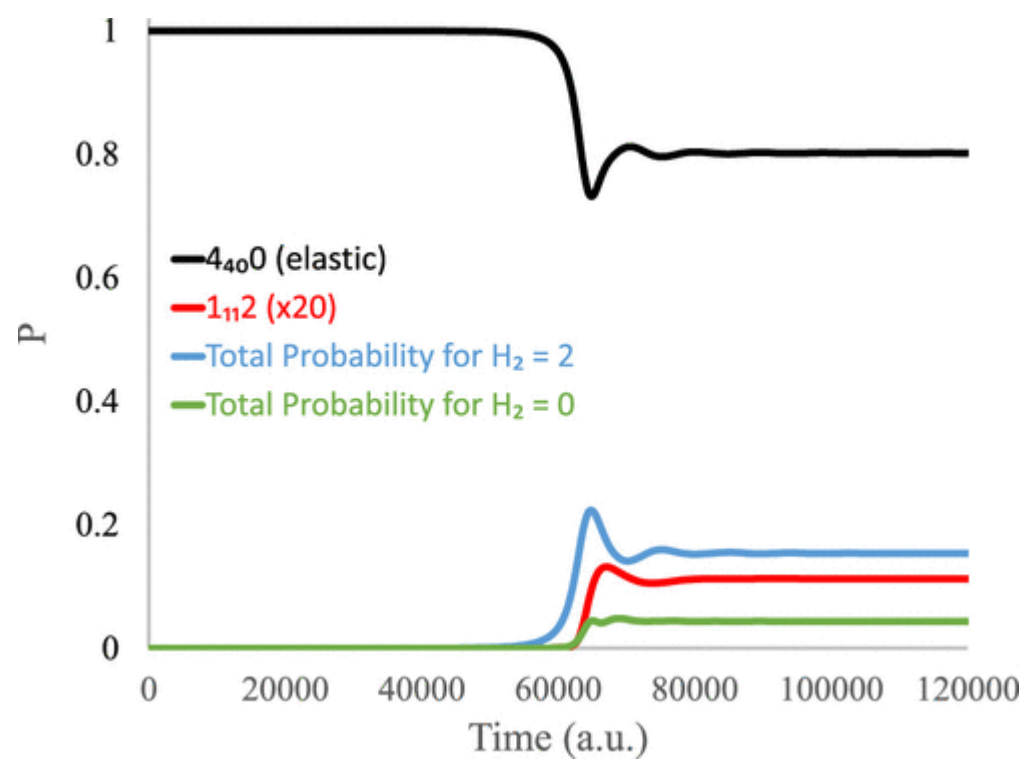


Figure 6. Time evolution of state probabilities along a typical MQCT trajectory (see text) that indicate a direct exchange of populations between the excited water state and the excited hydrogen state, a quasi-resonance energy exchange process $4_{40} 0 \rightarrow 1_{11}$.

We inspected the time evolution of all probability amplitudes, $a_{m n}(t)$, and computed the total probability for the excitation of hydrogen to $j_{2}=2$ (summed over all states of water molecule, namely, $0_{00} 2,1_{11} 2,2_{02} 2,2_{11} 2$, etc.) This dependence is shown in blue in Figure 6 , and we see that it is closer to the mirror image of the survival probability. The remaining small contribution is a probability for hydrogen to survive in the ground state $j_{2}=0$, while the water molecule is quenched and/or excited. This missing piece is plotted in Figure 6 in green. Now, we see that, in a time-dependent picture, all these energy exchange processes happen synchronously.

\section{Discussion}

\section{Numerical Speedup}

Now, let us address the question of a computational speedup of the MQCT method due to the adiabatic trajectory approximation, AT-MQCT. The overall speedup is very substantial, but it comes from two separate sources. The cost of running the first (adiabatic) step of the AT-MQCT calculations is insignificant since the size of the adiabatic basis is rather small. Associated numerical costs can be neglected for the sake of transparency.

One substantial contribution to the speedup comes from the fact that, in the second part of AT-MQCT calculations, we only need to propagate the quantum system of equations for the probability amplitudes, eq 5, instead of eqs 1-5 propagated together in the original CC-MQCT method. As explained above, when the basis size is large, the cost of computing the average gradient and torque in the right-hand sides of eqs 3 and 4 is dominant, and the overall cost associated with these two classical-like equations is approximately $65 \%$ (in the original version of the MQCT method). However, this is not anymore required in the AT-MQCT, so the numerical gain of the approximate method is expected to be on the order of $\times 3$. We carefully monitored the numerical cost of the full-coupled CCMQCT (the original method) and AT-MQCT calculations (new approximate method) and confirmed that the acceleration close to the factor of three is indeed achieved in our code.

The second contribution to the speedup comes from the efficient adiabatic step-size predictor used to accelerate integration of the quantum-like system of equations, eq 5 , during the second step of the ATMQCT calculations. The associated computational speed-up can be obtained by determining the number of time steps taken by the older version of the integrator with a constant step-size and the new version with an adjustable step-size predictor and then dividing one by the other (note that both versions use the RK4 integrator with the same numerical cost of one time step). We collected such data in a series of the numerical tests, making sure that both the constant step-size and the variable step-size calculations converge to about the same accuracy. We found that the numerical speed-up is sensitive to the collision energy, and these data are presented in Table 1 . They indicate that the numerical gain associated with the adiabatic step-size predictor is very substantial. The largest acceleration, by a factor close to $\times 70$, is found at intermediate collision energies. At higher collision energies, this is reduced to a factor of about $\times 33$. 


\begin{tabular}{|l|l|}
\hline $\boldsymbol{U}\left(\mathbf{c m}^{-1}\right)$ & speedup \\
\hline 20 & 22.5 \\
\hline 30 & 25.8 \\
\hline 50 & 32.2 \\
\hline 200 & 49.5 \\
\hline 500 & 69.5 \\
\hline 700 & 63.7 \\
\hline 2000 & 48.0 \\
\hline 7000 & 33.1 \\
\hline
\end{tabular}

Overall, taking into account both sources of the computational speedup, the AT-MQCT calculations appear to be faster than the original MQCT method by a factor ranging from about $\times 200$ at intermediate scattering energies to about $\times 100$ at high scattering energies. Since MQCT is more reliable at high collision energies and the intended use of this approximate method is at high collision energies, we can deduce, as a take-home message, that the adiabatic trajectory approximation within the MQCT framework reduces the numerical cost of scattering calculations by 2 orders of magnitude.

This makes many previously expensive calculations quite affordable now. As an example, we want to report the cost of the two runs we made during this work at collision energies of 500 and $2000 \mathrm{~cm}^{-}$ ${ }^{1}$ (the initial rotational state was $2{ }_{11} 2$ in both cases). These calculations were performed on a Cori computer at the NERSC(57) using only one node with 32 processors (2.3 GHz Intel Xeon). The wall-clock time of the run at $U=500 \mathrm{~cm}^{-1}$ was about $9 \mathrm{~min}$, while at $U=2000 \mathrm{~cm}^{-1}$, it was about $23 \mathrm{~min}$. It should also be taken into account that, out of this CPU time, only about $60 \%$ was spent on the actual propagation of the MQCT equations of motion, while about $40 \%$ is an overhead of the code associated with distribution of the initial data (array allocation, etc.) and collection of the final results (including the MPI barrier). These costs can be substantially reduced by code optimization. It should also be kept in mind that the cost of MQCT calculations depends on the level of rotational excitation since the number of initial states to be sampled and the basis size itself both depend on $j_{12}$. The corresponding cost scales linearly as $2 j_{12}+1$.

\section{On a Hierarchy of the Approximate MQCT Methods}

The adiabatic trajectory approximation considered here, AT-MQCT, can be thought of as one member of the family of approximate solutions of the MQCT equations of motion (eqs 1-5). Indeed, in the second step of AT-MQCT calculations, the quantum-like system of coupled equations (eq 5) for the probability amplitudes, $a_{m n}(t)$, is propagated, decoupled from the classical-like trajectory, and precomputed in an independent first run. However, using the same overall two-step approach, we could explore various options for pre-computing the MQCT trajectory. Indeed, it does not necessarily have to be an adiabatic single-state trajectory. One can include more than one rotational state in the basis during the first run! Of course, this would increase the numerical cost of the first run but hopefully not by much. 
If we have a good physical intuition about what quantum states need to be included into the molecular basis to pre-compute the trajectory, we could include these states into the basis of the first run right away. Then, in a sense, we would operate with two basis sizes: one relatively small (reduced) basis for pre-computing the mean-field trajectories and recording the $R(t), \dot{\Phi}(t)$, and $V(t)$ dependencies and a second (complete) basis for propagating the probability amplitudes, $a_{m n}(t)$, and computing the stateto-state transition cross sections. One can recall that similar algorithms of varying the basis size between different stages of calculations are routinely used in the electronic structure theory.

If we do not know a priori what molecular states need to be included into the trajectory calculations, we can either experiment by including several states energetically close to the initial state (since transition probabilities normally decrease as $\varepsilon_{n}^{n \prime}$ increases), or we could simply run the adiabatic ATMQCT calculations (both first and second steps since their cost is relatively low) and analyze their results to identify the most important molecular states. Then, the basis for the mean-field trajectory could be increased by including these states, and a new set of trajectory calculations is re-run with a meaningful (still reduced) basis followed by the final propagation of the quantum eq 5 to determine $a_{m n}(t)$ and calculate the cross sections. If needed, this procedure could be repeated in an iterative fashion. Again, the exclusion of unnecessary states from the basis is often done in the electronic structure calculations.

In order to demonstrate this general approach, we carried out some of such iterative calculations. The results are presented in Figure 7 for the initial ground state of the $\mathrm{H}_{2} \mathrm{O}+\mathrm{H}_{2}$ system, $\mathrm{O}_{00} \mathrm{O}$, with 20 rotational states in the basis, same as in the calculations reported in Figure 1 . The collison energy here was $U=200 \mathrm{~cm}^{-1}$. Three sets of calculations are compared in Figure 7. Green bars correspond to the AT-MQCT method with only one rotational state included into the basis to pre-compute the trajectory info (the adiabatic trajectory approach). From these calculations, one can see that, in addition to the initial ground state \#1, six excited rotational states gain significant populations during the collision. Large cross sections are found for states \#2, 3, 5, 6, 8, and 15. In particular, state \#15 at high energy corresponds to the excitation of $\mathrm{H}_{2}$ to the $j_{2}=2$ state. It makes sense to include these six excited states for pre-computing the mean-field trajectory data. The results of such calculations with seven rotational states in the basis (expanded relative to the adiabatic trajectory case but still reduced relative to the overall basis of 20 states) are presented in Figure 7 by blue bars. They indicate a consistent trend toward the results of the full-coupled CC-MQCT calculations shown by red bars (where all states are included, and all equations of motion are propagated together in a coupled fashion).

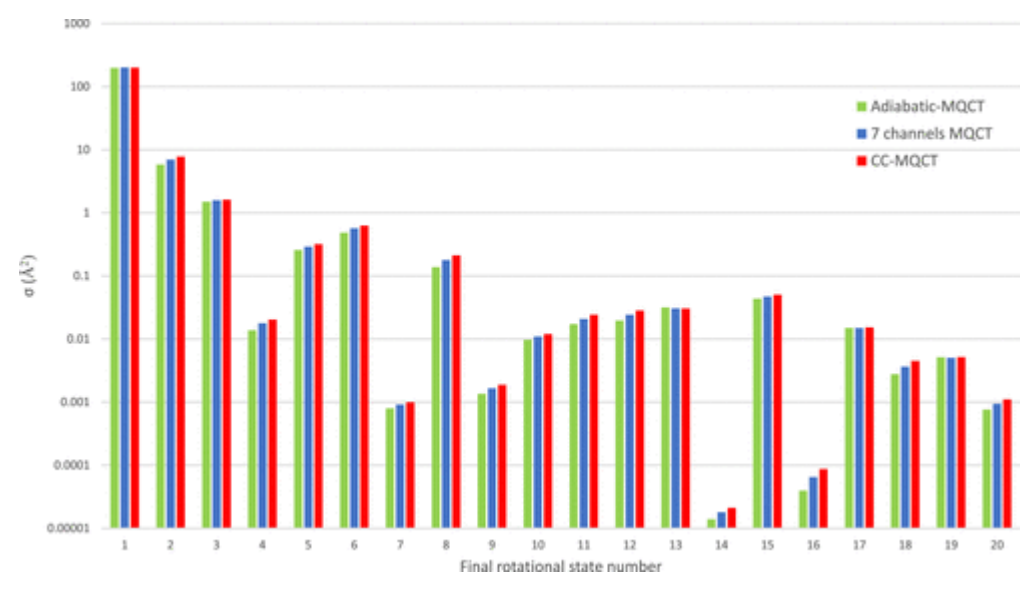


Figure 7. Comparison of results of the single-state AT-MQCT method (green bars) with calculations where seven rotational states are included in the trajectory prediction (blue bars) and with the full-coupled CC-MQCT calculations in a basis of twenty rotational states (red bars). The initial state is the ground state $0_{00} \mathrm{O}$ of the $\mathrm{H}_{2} \mathrm{O}+$ $\mathrm{H}_{2}$ system. The collision energy is $200 \mathrm{~cm}^{-1}$. Final states are listed along the horizontal axis. The values of collision cross sections are plotted along the vertical axis using the log scale.

Therefore, we conclude that, in principle, one could employ a hierarchy of the approximate treatments that converge monotonically to the most accurate solution, and thus one can always achieve the desired degree of accuracy if needed. The single-state AT-MQCT method is a limiting case in this hierarchy, which is also the fastest to run and the simplest to implement. Moreover, the results of calculations presented in this work indicate that AT-MQCT is accurate enough for many applications and thus is a good starting point of exploration of any molecular system. It may become the "work horse" within the MQCT series of methods.

We also want to note that a similar hierarchy of approximations can be formulated without decoupling the classical-like equations of motion (eqs 1-4) from the quantum-like equations for probability amplitudes (eq 5). In this case, we would only talk about truncation of the triple sum in the right-hand sides of eq 3 for the average gradient and eq 4 for the average torque. In a limiting case, the summation would be restricted to the $m$ states of one rotational channel that corresponds to the initial state of collision partners (a.k.a. adiabatic), or one could expand this summation to include more rotational states into the estimate of the right-hand sides of eqs 3 and 4 , and, in principle, one could do this iteratively, monitoring convergence. We tried some of such calculations too and found that they also work and give some computational advantage relative to the full-coupled CC-MQCT. However, without decoupling, it is harder to take full advantage of the computational speedup enabled by the adiabatic step-size predictor.

Therefore, we have chosen to stick with the decoupled AT-MQCT method. This option is now coded and will be made available to users in the new release of the program called MQCT 2020 that will happen later this year.

\section{Conclusions}

In this paper, we reviewed the formalism of the mixed quantum/classical theory (MQCT) for calculations of molecular inelastic scattering and proposed a hierarchy of the approximate methods for solutions of the corresponding equations of motion. In the simplest limiting case, which we named ATMQCT, we decouple the classical-like equations of motion from the quantum like equations for the propagation of probability amplitudes. The MQCT trajectories are pre-computed in the first step of calculations where the driving forces are determined by the potential energy surface of the entrance channel alone, which is an adiabatic trajectory (AT) approach. The quantum state-to-state transition probabilities are computed during the second step with an expanded basis and using the precomputed trajectory information for an efficient variable step-size integration.

This method was applied to a real system of $\mathrm{H}_{2} \mathrm{O}+\mathrm{H}_{2}$, and it was found that a very significant acceleration, by 2 orders of magnitude, is obtained at high collision energies. The results of the approximate propagation scheme are still rather accurate, as determined by comparison against more rigorous CC-MQCT calculations in which the quantum and classical equations of motion are propagated 
together in a coupled fashion but also against the full-quantum CC calculations from the literature (using the same potential energy surface).

Therefore, we conclude that our new AT-MQCT method represents a practical option for solving the inelastic scattering problem at high collision energies and for complex molecular collision partners (heavy molecules with many internal states and heavy collision partners), when the standard fullquantum calculations become computationally unaffordable. The next step would be to apply this theory to much more demanding molecular systems and scattering processes, such as $\mathrm{H}_{2} \mathrm{O}+\mathrm{H}_{2} \mathrm{O}$ rotationally inelastic scattering. Another avenue of the research could be to replace the generic Runge-Kutta integrator by another more specific routine, such as a velocity-Verlet,(58) a preconditioned Lanczosh, $(59)$ or a symplectic $(60,61)$ integrator, in conjunction with the adiabatic stepsize predictor.

The authors declare no competing financial interest.

\section{Notes}

The authors declare no competing financial interest.

\section{Acknowledgments}

This research was supported by the NASA Astrophysics Program, grant no. 15-APRA15-0115. B.M. acknowledges support of the Eisch Fellowship at Marquette. We used resources of the National Energy Research Scientific Computing Center, which is supported by the Office of Science of the U.S. Department of Energy under contract no. DE-AC02-5CH11231. Marie-Lise Dubernet is gratefully acknowledged for sharing the PES of $\mathrm{H}_{2} \mathrm{O}+\mathrm{H}_{2}$ and her full quantum results.

\section{References}

1. Sharples, T. R.; Leng, J. G.; Luxford, T. F. M.; McKendrick, K. G.; Jambrina, P. G.; Aoiz, F. J.; Chandler, D. W.; Costen, M. L. Non-Intuitive Rotational Reorientation in Collisions of NO $\left(A^{2} \Sigma^{+}\right)$with Ne from Direct Measurement of a Four-Vector Correlation. Nat.

Chem. 2018, 10, 1148-1153, DOI: 10.1038/s41557-018-0121-9

2. Goldberg, N. T.; Zhang, J.; Koszinowski, K.; Bouakline, F.; Althorpe, S. C.; Zare, R. N. Vibrationally Inelastic $\mathrm{H}+\mathrm{D}_{2}$ Collisions Are Forward-Scattered. Proc. Natl. Acad.

Sci. 2008, 105, 18194- 18199, DOI: 10.1073/pnas.0807942105

3. Faure, A.; Dagdigian, P. J.; Rist, C.; Dawes, R.; Quintas-Sánchez, E.; Lique, F.; Hochlaf, M. Interaction of Chiral Propylene Oxide $\left(\mathrm{CH}_{3} \mathrm{CHCH}_{2} \mathrm{O}\right)$ with Helium: Potential Energy Surface and Scattering Calculations. ACS Earth Space Chem. 2019, 3, 964-972, DOI: 10.1021/acsearthspacechem.9b00069

4. Ben Khalifa, M.; Quintas-Sánchez, E.; Dawes, R.; Hammami, K.; Wiesenfeld, L. Rotational Quenching of an Interstellar Gas Thermometer: $\mathrm{CH}_{3} \mathrm{CN}$...He Collisions. Phys. Chem. Chem. Phys. 2020, 22, 17494-17502, DOI: 10.1039/D0CP02985H

5. Denis-Alpizar, O.; Stoecklin, T.; Dutrey, A.; Guilloteau, S. Rotational Relaxation of $\mathrm{HCO}^{+}$and $\mathrm{DCO}^{+}$by Collision with $\mathrm{H}_{2}$. Mon. Not. R. Astron. Soc. 2020, 4276, DOI: 10.1093/mnras/staa2308 
6. Chen, J.; Li, J.; Bowman, J. M.; Guo, H. Energy Transfer between Vibrationally Excited Carbon Monoxide Based on a Highly Accurate Six-Dimensional Potential Energy Surface. J. Chem. Phys. 2020, 153, 54310, DOI: 10.1063/5.0015101

7. Chefdeville, S.; Kalugina, Y.; van de Meerakker, S. Y. T.; Naulin, C.; Lique, F.; Costes, M. Observation of Partial Wave Resonances in Low-Energy $\mathrm{O}_{2}-\mathrm{H}_{2}$ Inelastic Collisions. Science 2013, 341, 1094- 1096, DOI: 10.1126/science.1241395

8. Perreault, W. E.; Mukherjee, N.; Zare, R. N. Cold Quantum-Controlled Rotationally Inelastic Scattering of $\mathrm{HD}$ with $\mathrm{H}_{2}$ and $\mathrm{D}_{2}$ Reveals Collisional Partner Reorientation. Nat. Chem. 2018, 10, 561- 567, DOI: 10.1038/s41557-018-0028-5

9. Sun, Z.-F.; van Hemert, M. C.; Loreau, J.; van der Avoird, A.; Suits, A. G.; Parker, D. H. Molecular Square Dancing in CO-CO Collisions. Science 2020, 369, 307-309, DOI: 10.1126/science.aan2729

10. Lau, J. A.; Choudhury, A.; Chen, L.; Schwarzer, D.; Verma, V. B.; Wodtke, A. M. Observation of an Isomerizing Double-Well Quantum System in the Condensed Phase. Science 2020, 367, 175-178, DOI: 10.1126/science.aaz3407

11. Chen, L.; Lau, J. A.; Schwarzer, D.; Meyer, J.; Verma, V. B.; Wodtke, A. M. The Sommerfeld Ground-Wave Limit for a Molecule Adsorbed at a Surface. Science 2019, 363, 158- 161, DOI: 10.1126/science.aav4278

12. Chang, H.-C.; Ewing, G. E. Infrared Fluorescence from a Monolayer of CO on $\mathrm{NaCl}$ (100). Phys. Rev. Lett. 1990, 65, 2125, DOI: 10.1103/PhysRevLett.65.2125

13. Jiang, B.; Yang, M.; Xie, D.; Guo, H. Quantum Dynamics of Polyatomic Dissociative Chemisorption on Transition Metal Surfaces: Mode Specificity and Bond Selectivity. Chem. Soc. Rev. 2016, 45, 3621-3640, DOI: 10.1039/C5CS00360A

14. Jiang, B.; Guo, H. Dynamics in Reactions on Metal Surfaces: A Theoretical Perspective. J. Chem. Phys. 2019, 150, 180901, DOI: 10.1063/1.5096869

15. Alkoby, Y.; Chadwick, H.; Godsi, O.; Labiad, H.; Bergin, M.; Cantin, J. T.; Litvin, I.; Maniv, T.; Alexandrowicz, G. Setting Benchmarks for Modelling Gas--Surface Interactions Using Coherent Control of Rotational Orientation States. Nat. Commun. 2020, 11, 1-8, DOI: 10.1038/s41467-020-16930-1

16. Rui, J.; Yang, H.; Liu, L.; Zhang, D.-C.; Liu, Y.-X.; Nan, J.; Chen, Y.-A.; Zhao, B.; Pan, J.W. Controlled State-to-State Atom-Exchange Reaction in an Ultracold Atom--Dimer Mixture. Nat. Phys. 2017, 13, 699- 703, DOI: 10.1038/nphys4095

17. Morita, M.; Krems, R. V.; Tscherbul, T. V. Universal Probability Distributions of Scattering Observables in Ultracold Molecular Collisions. Phys. Rev. Lett. 2019, 123, 13401, DOI: 10.1103/PhysRevLett.123.013401

18. Kendrick, B. K.; Li, H.; Li, M.; Kotochigova, S.; Croft, J. F. E.; Balakrishnan, N. Non-Adiabatic Quantum Interference Effects and Chaoticity in the Ultracold $\mathrm{Li}+\mathrm{LiNa}-->\mathrm{Li}_{2}+\mathrm{Na}$ Reaction. arXiv Prepr. arXiv2006.15238; 2020.

19. Hazra, J.; Kendrick, B. K.; Balakrishnan, N. Geometric Phase Effects in Ultracold Hydrogen Exchange Reaction. J. Phys. B: At., Mol. Opt. Phys. 2016, 49, 194004, DOI: 10.1088/09534075/49/19/194004 
20. Wiesenfeld, L.; Faure, A.; Lique, F.; Dumouchel, N.; Feautrier, N.; Spielfiedel, A.; Scribano, Y. Analyzing Observations of Molecules in the ISM: Theoretical and Experimental Studies of Energy Transfer. EAS Publ. Ser. 2012, 58, 267- 270, DOI: 10.1051/eas/1258042

21. Roueff, E.; Lique, F. Molecular Excitation in the Interstellar Medium: Recent Advances in Collisional, Radiative, and Chemical Processes. Chem. Rev. 2013, 113, 8906-8938, DOI: 10.1021/cr400145a

22. Semenov, A.; Babikov, D. Mixed Quantum/Classical Approach for Description of Molecular Collisions in Astrophysical Environments. J. Phys. Chem. Lett. 2015, 6, 1854- 1858, DOI: 10.1021/acs.jpclett.5b00496

23. Faure, A.; Wiesenfeld, L.; Scribano, Y.; Ceccarelli, C. Rotational Excitation of Mono-and DoublyDeuterated Water by Hydrogen Molecules. Mon. Not. R. Astron. Soc. 2012, 420, 699- 704, DOI: 10.1111/j.1365-2966.2011.20081.x

24. Daniel, F.; Dubernet, M.-L.; Grosjean, A. Rotational Excitation of 45 Levels of Ortho/Para- $\mathrm{H}_{2} \mathrm{O}$ by Excited Ortho/Para- $\mathrm{H}_{2}$ from $5 \mathrm{~K}$ to $1500 \mathrm{~K}$ : State-to-State, Effective, and Thermalized Rate Coefficients. Astron. Astrophys. 2011, 536, A76, DOI: 10.1051/0004-6361/201118049

25. Wiesenfeld, L.; Faure, A. Ab Initio Computation of the Broadening of Water Rotational Lines by Molecular Hydrogen. Phys. Rev. A 2010, 82, 40702, DOI: 10.1103/PhysRevA.82.040702

26. Kendrick, B. K.; Hazra, J.; Balakrishnan, N. Geometric Phase Appears in the Ultracold Hydrogen Exchange Reaction. Phys. Rev. Lett. 2015, 115, 153201, DOI: 10.1103/PhysRevLett.115.153201

27. Mack, A.; Clark, T. K.; Forrey, R. C.; Balakrishnan, N.; Lee, T.-G.; Stancil, P. C. Cold He + $\mathrm{H}_{2}$ Collisions near Dissociation. Phys. Rev. A 2006, 74, 52718, DOI: 10.1103/PhysRevA.74.052718

28. Hutson, J. M.; Le Sueur, C. R. MOLSCAT: A Program for Non-Reactive Quantum Scattering Calculations on Atomic and Molecular Collisions. Comput. Phys.

Commun. 2019, 241, 9- 18, DOI: 10.1016/j.cpc.2019.02.014

29. Hutson, J. M.; Le Sueur, C. R. MOLSCAT, BOUND, and FIELD; Version 2020.0. 2020.

30. Alexander, M. H.; Manolopoulos, D. E.; Werner, H. J.; Follmeg, B. The HIBRIDON Package; 2014.

31. Semenov, A.; Mandal, B.; Babikov, D. MQCT: User-Ready Program for Calculations of Inelastic Scattering of Two Molecules. Comput. Phys. Commun. 2020, 107155

32. Bonnet, L. Semiclassical Initial Value Theory of Rotationally Inelastic Scattering: Some Remarks on the Phase Index in the Interaction Picture. J. Chem. Phys. 2018, 148, 194104, DOI: 10.1063/1.5024785

33. Loreau, J.; Faure, A.; Lique, F. Scattering of $\mathrm{CO}$ with $\mathrm{H}_{2} \mathrm{O}$ : Statistical and Classical Alternatives to Close-Coupling Calculations. J. Chem. Phys. 2018, 148, 244308, DOI: 10.1063/1.5036819

34. Semenov, A.; Babikov, D. Equivalence of the Ehrenfest Theorem and the Fluid-Rotor Model for Mixed Quantum/Classical Theory of Collisional Energy Transfer. J. Chem.

Phys. 2013, 138, 164110, DOI: 10.1063/1.4801430

35. Sur, S.; Ndengué, S. A.; Quintas-Sánchez, E.; Bop, C.; Lique, F.; Dawes, R. Rotationally Inelastic Scattering of $\mathrm{O}_{3}-$-Ar: State-to-State Rates with the Multiconfigurational Time Dependent Hartree Method. Phys. Chem. Chem. Phys. 2020, 22, 1869- 1880, DOI: 10.1039/C9CP06501F

36. Boursier, C.; Mandal, B.; Babikov, D.; Dubernet, M. L. New $\mathrm{H}_{2} \mathrm{O}-\mathrm{H}_{2} \mathrm{O}$ Collisional Rate Coefficients for Cometary Applications. Mon. Not. R. Astron. Soc. 2020, 498, 5489- 5497, DOI: 10.1093/mnras/staa2713 
37. Ivanov, M. V.; Babikov, D. Efficient Quantum-Classical Method for Computing Thermal Rate Constant of Recombination: Application to Ozone Formation. J. Chem.

Phys. 2012, 136, 184304, DOI: 10.1063/1.4711760

38. Babikov, D.; Semenov, A. Recent Advances in Development and Applications of the Mixed Quantum/Classical Theory for Inelastic Scattering. J. Phys. Chem. A 2015, 120, 319- 331, DOI: 10.1021/acs.jpca.5b09569

39. Semenov, A.; Babikov, D. Mixed Quantum/Classical Theory of Rotationally and Vibrationally Inelastic Scattering in Space-Fixed and Body-Fixed Reference Frames. J. Chem.

Phys. 2013, 139, 044306 DOI: 10.1063/1.4827256

40. Semenov, A.; Babikov, D. Mixed Quantum/Classical Calculations of Total and Differential Elastic and Rotationally Inelastic Scattering Cross Sections for Light and Heavy Reduced Masses in a Broad Range of Collision Energies. J. Chem. Phys. 2014, 140, 044306, DOI: 10.1063/1.4862409

41. Semenov, A.; Babikov, D. MQCT. I. Inelastic Scattering of Two Asymmetric-Top Rotors with Application to $\mathrm{H}_{2} \mathrm{O}+\mathrm{H}_{2} \mathrm{O}$. J. Phys. Chem. A 2017, 121, 4855, DOI: 10.1021/acs.jpca.7b03554

42. Semenov, A.; Babikov, D. Accurate Calculations of Rotationally Inelastic Scattering Cross Sections Using Mixed Quantum/Classical Theory. J. Phys. Chem. Lett. 2014, 5, 275- 278, DOI: 10.1021/jz402542w

43. Semenov, A.; Babikov, D. Inelastic Scattering of Identical Molecules within Framework of the Mixed Quantum/Classical Theory: Application to Rotational Excitations in $\mathrm{H}_{2}+\mathrm{H}_{2}$. J. Phys. Chem. A 2016, 120, 3861-3866, DOI: 10.1021/acs.jpca.6b04556

44. Semenov, A.; Babikov, D. Mixed Quantum/Classical Theory for Molecule-Molecule Inelastic Scattering: Derivations of Equations and Application to $\mathrm{N}_{2}+\mathrm{H}_{2}$ System. J. Phys. Chem. A 2015, 119, 12329-12338, DOI: 10.1021/acs.jpca.5b06812

45. Semenov, A.; Dubernet, M. L.; Babikov, D. Mixed Quantum/Classical Theory for Inelastic Scattering of Asymmetric-Top-Rotor + Atom in the Body-Fixed Reference Frame and Application to the $\mathrm{H}_{2} \mathrm{O}+\mathrm{He}$ System. J. Chem. Phys. 2014, 141, 114304, DOI: 10.1063/1.4895607

46. Ivanov, M. V.; Babikov, D. On Molecular Origin of Mass-Independent Fractionation of Oxygen Isotopes in the Ozone Forming Recombination Reaction. Proc. Natl. Acad.

Sci. 2013, 110, 17708-17713, DOI: 10.1073/pnas.1215464110

47. Mandal, B.; Semenov, A.; Babikov, D. Calculations of Differential Cross Sections Using Mixed Quantum / Classical Theory of Inelastic Scattering; 2018.

48. Billing, G. D. The Semiclassical Treatment of Molecular Roto/Vibrational Energy Transfer. Comput. Phys. Rep. 1984, 1, 239-296, DOI: 10.1016/0167-7977(84)90006-6

49. Billing, G. D. The Quantum Classical Theory; Oxford University Press: 2003.

50. Pack, R. T. Space-Fixed vs Body-Fixed Axes in Atom-Diatomic Molecule Scattering. Sudden Approximations. J. Chem. Phys. 1974, 60, 633-639, DOI: 10.1063/1.1681085

51. Pack, R. T. Coordinates for an Optimum CS Approximation in Reactive Scattering. Chem. Phys. Lett. 1984, 108, 333- 338, DOI: 10.1016/0009-2614(84)85201-X

52. McGuire, P.; Kouri, D. J. Quantum Mechanical Close Coupling Approach to Molecular Collisions. Jz-Conserving Coupled States Approximation. J. Chem. Phys. 1974, 60, 2488- 2499, DOI: $10.1063 / 1.1681388$ 
53. Kouri, D. J.; Goldflam, R.; Shimoni, Y. On the Coupled States and Infinite Order Sudden Approximations to the Body Frame Wavefunction. J. Chem. Phys. 1977, 67, 4534- 4544, DOI: 10.1063/1.434594

54. Heil, T. G.; Green, S.; Kouri, D. J. The Coupled States Approximation for Scattering of Two Diatoms. J. Chem. Phys. 1978, 68, 2562-2583, DOI: 10.1063/1.436115

55. Press, W. H.; Teukolsky, S. A.; Flannery, B. P.; Vetterling, W. T. Numerical Recipes in Fortran 77: Volume 1, Volume 1 of Fortran Numerical Recipes: The Art of Scientific Computing; Cambridge university press: 1992.

56. Yang, C. H.; Sarma, G.; Parker, D. H.; Ter Meulen, J. J.; Wiesenfeld, L. State-to-State Differential and Relative Integral Cross Sections for Rotationally Inelastic Scattering of $\mathrm{H}_{2} \mathrm{O}$ by Hydrogen. J. Chem. Phys. 2011, 134, 204308, DOI: 10.1063/1.3589360

57. Cori System - NERSC Documentation .

58. Kong, X.; Markmann, A.; Batista, V. S. Time-Sliced Thawed Gaussian Propagation Method for Simulations of Quantum Dynamics. J. Phys. Chem. A 2016, 120, 3260-3269, DOI: 10.1021/acs.jpca.5b12192

59. Stancil, P. C.; You, H.; Cook, A.; Sornborger, A. T.; Geller, M. R. Towards Quantum Simulation of Chemical Dynamics with Prethreshold Superconducting Qubits. arXiv Prepr. arXiv1602.00063; 2016.

60. Gray, S. K.; Manolopoulos, D. E. Symplectic Integrators Tailored to the Time-Dependent Schrödinger Equation. J. Chem. Phys. 1996, 104, 7099- 7112, DOI: 10.1063/1.471428

61. Manolopoulos, D. E.; Gray, S. K. Symplectic Integrators for the Multichannel Schrödinger Equation. J. Chem. Phys. 1995, 102, 9214- 9227, DOI: 10.1063/1.468871 